PROCEEDINGS OF THE

AMERICAN MATHEMATICAL SOCIETY

Volume 129, Number 2, Pages 617-620

S 0002-9939(00)05585-

Article electronically published on September 20, 2000

\title{
ON THE DIMENSION OF A HOMEOMORPHISM GROUP
}

\author{
BEVERLY L. BRECHNER AND KAZUHIRO KAWAMURA \\ (Communicated by Alan Dow)
}

\begin{abstract}
We prove that the homeomorphism group of each one of a collection of continua constructed in a paper by the first author (Trans. Amer. Math. Soc. 121 (1966), 516-548) is one dimensional. This answers a question posed in that paper.
\end{abstract}

\section{INTRODUCTION}

For a compact metric space $X, G(X)$ denotes the space of all autohomeomorphisms on $X$ with the compact-open topology. It is a separable completely metrizable topological group. When $X$ is a topological manifold, the space $G(X)$ is infinite dimensional, while there exists a compact metrizable space $Y$ with $G(Y)=\left\{i d_{Y}\right\}$. Also it is known that the homeomorphism groups of the Sierpinski curve, as well as the universal Menger compactum $\mu^{n}$ ([1], [2]), are totally disconnected and exactly one-dimensional. (The proof of total disconnectivity for $\mu^{n}$ is similar to the proof for $\mu^{1}$ in $\left[3\right.$. The proof of nonzero dimensionality is the same as the proof for $\mu^{1}$ in [3], using Theorem 3.2.2 of Bestvina 2] which says that each $\mu^{n}, n \geq 1$, is strongly locally homogeneous.)

In an attempt to construct a compact connected metric space which has a homeomorphism group of finite, but at least two dimensions, the first author constructed in [3] compact connected metric spaces $M_{n}$, for all $n \geq 1$, with the following property: there exist compact metric spaces $L_{1}, L_{2}, \ldots, L_{n}, \ldots$ such that

1. $G\left(M_{n}\right)$ is topologically isomorphic to the product $\prod_{i=1}^{n} G\left(L_{i}\right)$.

2. Each $G\left(L_{i}\right)$ is totally disconnected and one-dimensional. Hence $G\left(M_{n}\right)$ is at most $n$-dimensional.

She asked whether the dimension of $G\left(M_{n}\right)$ is indeed $n$ or not. The purpose of this note is to prove that $\operatorname{dim} G\left(M_{n}\right)=1$. Throughout, a continuum means a compact connected metric space.

\section{BACKGROUND}

2.1. The continua $M_{n}$. Each compact metric space $L_{i}$ above is obtained by a single type of construction which is briefly described below. (A dendroid is a nondegenerate continuum $X$ such that each pair of distinct points $x, y \in X$ is

Received by the editors June 6, 1998 and, in revised form, May 8, 1999.

1991 Mathematics Subject Classification. Primary 54F45, 54G20; Secondary 54H15, 54H20.

Key words and phrases. Homeomorphism group, dimension, Menger continua. 
contained in a unique continuum $[x, y]$ which is minimal with respect to containing $x, y .[x, y]$ must be an arc, when $X$ is metric.)

There exists an infinite collection $\left\{D_{j}\right\}_{j=1}^{\infty}$ of locally connected dendroids such that $G\left(D_{j}\right)=\{i d\}$. Take an increasing sequence $\left\{p_{j}\right\}_{j=1}^{\infty}$ of primes greater than two. For a fixed $j$, dendroid $D_{j}$, and prime $p_{j}$, the construction below produces the compactum, $K=L_{j}$.

Fix $j$. Take two distinct end points $a_{j}$ and $b_{j}$ of $D_{j}$ and take a continuous surjection $f_{j}: D_{j} \rightarrow[0,1]$ such that $f_{j}^{-1}(0)=a_{j}$ and $f_{j}^{-1}(1)=b_{j}$. Let $E_{j}$ be the continuum obtained from $D_{j}$ by identifying $a_{j}$ and $b_{j}$, let $\phi_{j}$ be this identification map, and let $g_{j}: E_{j} \rightarrow S_{j}$ be the induced map from $E_{j}$ onto a simple closed curve $S_{j}$. Let $v_{j}=g_{j}\left(\phi_{j}\left(a_{j}\right)\right)=g_{j}\left(\phi_{j}\left(b_{j}\right)\right)$.

Let $\pi_{j_{i}}: S_{j_{i}} \rightarrow S_{j}$ be the $\left(p_{j}\right)^{i}$-fold covering of the simple closed curve to itself. Let $K_{j_{i}}$ be the fibre product of the maps $\pi_{j_{i}}$ and $g_{j}$ and let $h_{j_{i}}: K_{j_{i}} \rightarrow S_{j_{i}}$ be the projection of $K_{j_{i}}$ onto the $\left(p_{j}\right)^{i}$-fold cover of $S_{j}$. The points $h_{j_{i}}^{-1} \pi_{j_{i}}^{-1}\left(v_{j}\right)$ are called the vertices of $K_{j_{i}}$. The sequence $\left\{S_{j_{i}}\right\}_{i=1}^{\infty}$ can be identified with a sequence of concentric circles centered at the origin $o$ of the plane converging to the unit circle $T$ centered at $o$.

It is easy to see that $G\left(K_{j_{i}}\right)=\mathbf{Z}_{\left(p_{j}\right)^{i}}$, the cyclic group of order $p_{j}^{i}$, generated by a rotation $r_{j_{i}}$. The topological sum $\bigoplus K_{j_{i}}$ is mapped onto the union $\bigcup_{i=1}^{\infty} S_{j_{i}}$ by $\bigoplus h_{j_{i}}$ and hence compactified by adding the unit circle $T$ in the obvious way. The resulting compactum is denoted by $K$. The compactum $K$ is realized as a subset of the plane and what is important to us is the following property:

(*) For each homeomorphism $h \in G(K)$ and for each $i$, the restriction $h \mid K_{j_{i}}$ is a plane rotation $r_{j_{i}}^{k}\left(=k\right.$-fold iteration of $\left.r_{j_{i}}\right)$ for some $k$.

Roughly speaking, for a fixed prime $p$, one may think of a sequence of concentric, locally connected, "circular dendroids", $\left\{D_{i}\right\}_{i=1}^{\infty}$, the $i$ th one $D_{i}$ being the union of $p^{i}$ dendroids, and admitting exactly $p^{i}$ homeomorphisms, and constructed so that this sequence converges to the unit circle $T=T_{1}$. Then for $n=1$, the compactum is

$$
K=K_{1}=T_{1} \cup \bigcup_{i=1}^{\infty} D_{i} .
$$

For $n \geq 2$, one may obtain compacta $K_{n}$ by generalizing this construction, using limit tori $T_{n}$, instead of the circle $T=T_{1}$, and using primes $p_{1}, \ldots, p_{n}$ in the $n$ product directions, respectively. For $n=2$, it looks like a sequence of barbed wire grids converging to a limit torus. The continua $M_{n}$ are obtained from the $K_{n}$ 's by taking a cone over the vertices and limit tori, but replacing the free arcs of the cone by locally connected dendroids, each admitting exactly one homeomorphism. Then $G\left(M_{n}\right) \cong G\left(K_{n}\right)$, both topologically and algebraically.

For more detail, see [3], p. 540. In the following, we let $d$ denote the restriction of the standard metric of the plane to $K$.

2.2. Almost zero dimensional spaces. The key notion for our argument is the almost zero dimensionality [6]. A separable metrizable space $X$ is said to be almost zero dimensional if it admits a countable base $\mathcal{B}$ with the following property:

$\left.{ }^{* *}\right)$ For each pair $B_{1}, B_{2} \in \mathcal{B}$ with $\operatorname{cl}\left(B_{1}\right) \cap \operatorname{cl}\left(B_{2}\right)=\emptyset$, there exists a clopen set $G$ such that $B_{1} \subset G$ and $B_{2} \subset X \backslash G$.

Theorem 2.1 ([6], [5]). Each almost zero-dimensional separable metrizable space is at most one-dimensional. 


\section{MAIN THEOREM}

The following lemma is immediate from the definition of almost zero dimensional.

Lemma 3.1. Let $\left\{X_{i} \mid i=1,2,3, \ldots\right\}$ be a countable collection of almost zero dimensional separable metrizable spaces. Then the product $\prod_{i=1}^{\infty} X_{i}$ is almost zero dimensional.

It follows from the above that, for the proof of main theorem, it suffices to prove the following.

Theorem 3.2. Let $K$ be the compact metrizable space constructed in Section 2. Then the space $G(K)$ is almost zero dimensional.

Proof. The sup metric on $G(K)$ induced by the metric $d$ is also denoted by $d$ for simplicity. The closed $\varepsilon$-ball at $f \in G(K)$ is denoted by $\hat{B}(f, \varepsilon)$. By Theorem 4 of [6], it suffices to prove the following claim:

for each $f \notin \hat{B}(i d, \varepsilon)$, there exists a clopen set $V \subset G(K) \backslash \hat{B}(i d, \varepsilon)$ such that $f \in V$.

Suppose that $d(f, i d)=\varepsilon+\delta$ for $\delta>0$. There exists a positive integer $i$ such that $d\left(f \mid K_{j_{i}}, i d\right)>\varepsilon$. Since $f \mid K_{j_{i}}$ is a planar rotation (see the property $\left({ }^{*}\right)$ ), there exists a vertex $x \in K_{j_{i}}$ such that $d(f(x), x)>\varepsilon$. Also by the property $\left({ }^{*}\right), f \mid K_{j_{i}}=r_{j_{i}}^{k}$ for some $k$.

Let $i_{0}=\min \left\{|k| \mid d\left(r_{j_{i}}^{k}, i d\right)>\varepsilon\right\}$ and define $U=\left\{h \in G(K)|h| K_{j_{i}}=r_{j_{i}}^{k}\right.$ for some $k$, with $\left.|k| \leq i_{0}-1\right\}$ and $V=\left\{h \in G(K)|h| K_{j_{i}}=r_{j_{i}}^{k}\right.$ for some $k$, with $\left.|k| \geq i_{0}\right\}$. It is easy to see that $U$ and $V$ are disjoint clopen sets such that $G(K)=U \cup V$. Further $\hat{B}(i d, \varepsilon) \subset U$ and $f \in V$. This completes the proof.

Our main theorem now can be stated as follows:

Theorem 3.3. The space $G\left(M_{n}\right)$ is almost zero dimensional and hence $\operatorname{dim} G\left(M_{n}\right)$ $=1$.

The proof follows immediately from Theorem 3.2 above.

\section{Open PROBLEM}

The problem raised in [3], namely whether there exists a compact metric space, or metric continuum, $K$, such that $1<\operatorname{dim} G(K)<\infty$, remains open.

We note that in [4], Keesling and Wilson construct examples, $X_{n}$, of $(n-1)$ dimensional, connected, locally connected subgroups of $R^{n}$, whose homeomorphism groups are also of dimension $(n-1)$. Thus, they establish the existence of spaces whose homeomorphism groups are of dimension $n$, for all $n \geq 1$. However, the spaces are neither compact nor even locally compact.

\section{REFERENCES}

[1] Mladen Bestvina, Characterizing k-dimensional universal Menger compacta, Bull. Amer. Math. Soc. (N.S.) 11 (1984), no.2, 369-370. MR 86g:54047

[2] Mladen Bestvina, Characterizing $k$-dimensional universal Menger compacta, Memoirs Amer. Math. Soc 71 (1988), no. 380, vi+110 pp. MR 89g:54083

[3] B. L. Brechner, On the dimensions of certain spaces of homeomorphisms, Trans. Amer. Math. Soc. 121 (1966), 516-548. MR 32:4662

[4] J. E. Keesling and D. C. Wilson, An almost uniquely homogeneous subgroup of $R^{n}$, Topology and its Applications 22 (1986), 183-190. MR 87j:22002 
[5] M. Levin and R. Pol, A metric condition which implies dimension $\leq 1$, Proc. Amer. Math. Soc. 125 (1997), 269-273. MR 97e:54033

[6] L. G. Oversteegen and E. D. Tymchatyn, On the dimension of certain totally disconnected spaces, Proc. Amer. Math. Soc. 122 (1994), 885-891. MR 95b:54040

Department of Mathematics, University of Florida at Gainesville, Gainesville, FLORIDA 32611-8105

E-mail address: brechner@math.ufl.edu

Institute of Mathematics, University of Tsukuba, Tsukuba-shi, Ibaraki 305 Japan

E-mail address: kawamura@math.tsukuba.ac.jp 